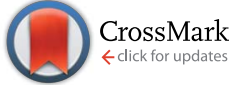

Cite this: RSC Adv., 2017, 7, 2550

Received 7th December 2016 Accepted 21st December 2016

DOI: 10.1039/c6ra27629f

www.rsc.org/advances

\title{
Large electrostrain with good temperature stability in sodium niobate based ceramics
}

\author{
Wenfeng Liu, * Daqi Zhao and Shengtao Li
}

A large electrostrain of $0.24 \%$ with a converse piezoelectric constant of $790 \mathrm{pm} \mathrm{V}^{-1}$ was obtained from the $\mathrm{LiSbO}_{3}$ and $\left(\mathrm{Bi}_{0.5} \mathrm{Na}_{0.5}\right)\left(\mathrm{Zr}_{0.9} \mathrm{Sn}_{0.1}\right) \mathrm{O}_{3}$ modified $\mathrm{KNbO}_{3}$ ceramics. The electrostrain showed good temperature stability with vibration within $3 \%$ in the range from room temperature to $200{ }^{\circ} \mathrm{C}$. It can even rival the current working horse PZT4. Besides, the present ceramics exhibited a large $d_{33}$ of $207 \mathrm{pC} \mathrm{N}{ }^{-1}$. The enhanced piezoelectric properties were achieved by pinching the phase with different symmetries together, experimentally by doping. Here the isovalent additive $\mathrm{LiSbO}_{3}$ was employed to lower the tetragonal to orthorhombic phase transition temperature and to raise the orthorhombic to rhombohedral phase transition temperature, i.e. pinch the three ferroelectric phases together. Meanwhile $\left(\mathrm{Bi}_{0.5} \mathrm{Na}_{0.5}\right)\left(\mathrm{Zr}_{0.9} \mathrm{Sn}_{0.1}\right) \mathrm{O}_{3}$ was used as the aliovalent doping to lower the $T_{\mathrm{c}}$. It consequently resulted in a decrease of polarization anisotropy between phases with different symmetries and thus led to a large piezoelectric performance.

\section{Introduction}

Piezoelectric materials exhibit a shape change to a predefined state upon a change of the electric field. Such materials provide a good solution to the "quick and slim" actuator applications since they guarantee a fast response and their required driven field can be easily applied through very fine guide wires (compared with the bulky magnets required by magnetic-shapememory materials) $\cdot^{1-3}$ Many applications, typically the actuators, require large electrostrain under a high driving field, and at the present stage this market is dominated by $\mathrm{Pb}$-based materials in the form of the ceramics. ${ }^{4-6}$ Despite their outstanding performance, $\mathrm{Pb}$-based ferroelectrics are currently facing global restrictions for their toxicity. Besides, the materials have to suffer the variations by changing temperature, emerging from varying ambient conditions as well as from the generation of heat by the inner losses during operation of these devices; thus a significant effort should be directed to develop non- $\mathrm{Pb}$ substitutes with both large electrostrain and high temperature stability.

Lead zirconate-titanate (PZT) based ceramics, the most widely used piezoelectric ceramics for electro-shape-memory applications, typically show an electrostrain of $0.15 \%$ under the electric field of $2 \mathrm{kV} \mathrm{mm}^{-1}$. Meanwhile it exhibits the fluctuation in electrostrain of $15 \%$ in the temperature range from room temperature to $200{ }^{\circ} \mathrm{C} .{ }^{7}$ On the other hand, the lead-free candidates show the inferior property. (BiNa)TiO ${ }_{3}$-based

State Key Laboratory of Electrical Institution and Power Equipment, School of Electrical Engineering, Xi'an Jiaotong University, Xi'an 710049, China. E-mail: liuwenfeng@xjtu.edu.cn ceramics, as the most promising candidate, can achieve the electrostrain of $0.4 \%$. However, the high driving electric field over $8 \mathrm{kV} \mathrm{mm}{ }^{-1}$ and the large nonlinearity with big strain hysteresis restrict its applications. ${ }^{8} \mathrm{BaTiO}_{3}$ ceramic material containing $\mathrm{Zr}$ additive exhibited electrostrain of $0.18 \%$ at $4 \mathrm{kV}$ $\mathrm{mm}^{-1},{ }^{9}$ and similar results were found for $(\mathrm{BaSr}) \mathrm{TiO}_{3}$ ceramics containing Mn additive with $0.12-0.15 \%$ electrostriction at $3 \mathrm{kV}$ $\mathrm{mm}^{-1}$. While the main flaw in $\mathrm{BaTiO}_{3}$ based piezoelectric is the low Curie temperature $\left(T_{\mathrm{c}}\right) ;^{10,11}$ the Curie temperature of pure $\mathrm{BaTiO}_{3}$ is only $130{ }^{\circ} \mathrm{C}$, and additives reduce it further. Thus $\mathrm{BaTiO}_{3}$ based materials can therefore only be used within a narrow range of temperatures (say, $50{ }^{\circ} \mathrm{C}$ ) in the vicinity of room temperature. In addition, extensive attention is focused on $(\mathrm{K}, \mathrm{Na}) \mathrm{NbO}_{3}$ based ceramics since the breakthrough made by Saito in $2004^{12}$ and recently by Wu JG. ${ }^{13-19}$ CZ5 $\left(0.95\left(\mathrm{Na}_{0.49^{-}}\right.\right.$ $\left.\left.\mathrm{K}_{0.49} \mathrm{Li}_{0.02}\right)\left(\mathrm{Nb}_{0.8} \mathrm{Ta}_{0.2}\right) \mathrm{O}_{3}-0.05 \mathrm{CaZrO}_{3}\right)$ reported by Wang $\mathrm{Ke},{ }^{20}$ as one of the most promising lead-free substitute, yielded the electrostrain of $0.16 \%$ at room temperature with variation less than $10 \%$ from room temperature to $175^{\circ} \mathrm{C}$.

In the present paper, we report a promising KNN-based ceramics with even enhanced electrostrain of $0.24 \%$ under 3 $\mathrm{kV} \mathrm{mm}^{-1}$, which can even rival the current working horse PZT4. Meanwhile such ceramics exhibit remarkable temperature stability of $3 \%$ in the temperature range from room temperature to $200{ }^{\circ} \mathrm{C}$. The enhanced electrostrain performance is discussed based on the coexistence of multi-phases, which contributes to the flat polarization rotation energy landscape.

Previous study pointed out that a tetragonal (T)-orthorhombic (O)-rhombohedral (R) phase coexistence gives totally flat polarization rotation energy landscape, while $\mathrm{T}-\mathrm{O}$ or $\mathrm{O}-\mathrm{R}$ single phase boundary has relatively large energy barriers. It is 
highly possible for (K,Na) $\mathrm{NbO}_{3}$ based ceramics, who has a $\mathrm{T}$ to $\mathrm{O}$ to $\mathrm{R}$ ferroelectric transition sequence originally. This can be achieved by doping. Here we chose $\mathrm{LiSbO}_{3}$ to lower T-O transition temperature and to rise $\mathrm{O}-\mathrm{R}$ transition temperature, i.e. pinch the three ferroelectric phases together. This will create the quadruple point, which may give more polarization instabilities as well as phase similarities and consequently decrease the polarization anisotropy and energy barriers for both polarization rotation (intrinsic contribution) and domain wall motion (extrinsic contribution). ${ }^{21-26}$ On the other hand, we chose $\left(\mathrm{Bi}_{0.5} \mathrm{Na}_{0.5}\right)\left(\mathrm{Zr}_{0.9} \mathrm{Sn}_{0.1}\right) \mathrm{O}_{3}$ as the aliovalent doping to lower the $T_{\mathrm{c}} \cdot{ }^{27,28}$ According to Landau theory of phase transition, when temperature is close to $T_{\mathrm{c}}$, the energy barrier for polarization switching decreases and may benefit the enhanced property at room temperature.

\section{Experimental}

The designed sample ceramic $0.96\left(0.95\left(\mathrm{~K}_{0.48} \mathrm{Na}_{0.52}\right) \mathrm{NbO}_{3}\right.$ $\left.0.05 \mathrm{LiSbO}_{3}\right)-0.04\left(\mathrm{Bi}_{0.5} \mathrm{Na}_{0.5}\right)\left(\mathrm{Zr}_{0.9} \mathrm{Sr}_{0.1}\right) \mathrm{O}_{3}$ (abbreviated as $\mathrm{KNN}$ 5LS-4BNZS hereafter) were fabricated by the conventional solid state reaction method. Raw materials of $\mathrm{Na}_{2} \mathrm{CO}_{3}(99.8 \%)$, $\mathrm{K}_{2} \mathrm{CO}_{3}$ (99\%), $\mathrm{Li}_{2} \mathrm{CO}_{3}$ (99.9\%), $\mathrm{Sb}_{2} \mathrm{O}_{5}$ (99.99\%), $\mathrm{Nb}_{2} \mathrm{O}_{5}$ (99.5\%), $\mathrm{SnO}_{2}$ (99.9\%), $\mathrm{ZrO}_{2}$ (99\%), and $\mathrm{Bi}_{2} \mathrm{O}_{3}$ (99.999\%) were mixed according to a predetermined ratio with addition of deionized water and planetary ball mixed for $4 \mathrm{~h}$, which were then dried and calcined at $850{ }^{\circ} \mathrm{C}$ for $4 \mathrm{~h}$. After the granulation and tableting the specimens were sintered at $1165^{\circ} \mathrm{C}$ for $3 \mathrm{~h}$ in air.

The microstructure and grain-size were measured by SEM (SEM, KEYENCEVE-9800) and Nano Measure software developed by FDU. Dielectric property as a function of temperature (from $-200{ }^{\circ} \mathrm{C}$ to $350{ }^{\circ} \mathrm{C}$ ) were determined using two LCR testers (Agilent 4980a for $30{ }^{\circ} \mathrm{C}$ to $350{ }^{\circ} \mathrm{C}$ and 3532-50 HIOKI for $-200{ }^{\circ} \mathrm{C}$ to $30{ }^{\circ} \mathrm{C}$ ) at $10 \mathrm{kHz}$. Ferroelectric hysteresis loops and electrostrain curves were measured at temperature range from $20{ }^{\circ} \mathrm{C}$ to $200{ }^{\circ} \mathrm{C}$ using Radiant Workstation and MTI 2000 photic sensor. The frequency of the measurement was fixed at $10 \mathrm{~Hz}$. Piezoelectric constant $d_{33}$ was measured using a quasi-static $d_{33}$ meter (ZJ-3B, Institute of Acoustics, Chinese Academy of Sciences, China).

\section{Results and discussion}

Fig. 1(a) shows the temperature dependence of dielectric constant and loss tangent for the KNN-5LS-4BNZS ceramics. A dielectric maximum around 5100 under the frequency of $10 \mathrm{kHz}$ is achieved at $270{ }^{\circ} \mathrm{C}$, i.e. its $T_{\mathrm{c}}$. Here the broad peak indicating the diffusion phase transition region, which can be described by the full width at half maximum (FWHW) of $70^{\circ} \mathrm{C}$. These results indicate the existence of a diffuse phase transition in the present ceramic sample. ${ }^{29,30}$ Here, the long-range polar order is suppressed by substitution disorder, i.e. by doping extensive point defects. The quenched compositional disorder somehow prevents the normal transition into the phases with macroscopic ferroelectric state. Meanwhile, defect dipoles are formed by the substitution dopants; they act as the inherent force to a decrease of the transition temperature. ${ }^{31}$ Previous study

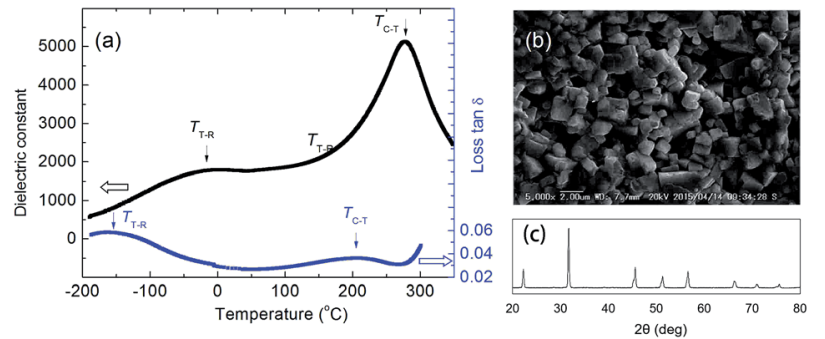

Fig. 1 (a) Temperature dependence of dielectric constant and loss tangent of the KNN-5LS-4BNZS ceramics. The broad peak at $270{ }^{\circ} \mathrm{C}$ indicated a diffusion phase transition. (b) SEM images and the grain size distribution of the KNN-5LS-4BNZS ceramics. (c) XRD patterns of the KNN-5LS-4BNZS ceramics.

pointed out that the $\mathrm{LiSbO}_{3}$ may effectively decrease the transition temperature from tetragonal to orthorhombic phase while increase the transition temperature from orthorhombic to rhombohedral phase, i.e. try to pinch all the ferroelectric transitions together by narrow the intermediate orthorhombic phase. ${ }^{13}$ Within the temperature region, manifested as the plateau in the temperature spectrum of the dielectric constant, multi phases may coexist. Accomplishing with the diffuse phase transition, the domain size always decreases, i.e. the population of domain wall enhances; it may enhance electrostrain which mainly originated from the extrinsic piezoelectric effect dominated by the domain wall motions. ${ }^{32}$ Fig. 1(b) shows the SEM images of the surface morphologies the ceramics. It indicates that the isotropic grains with the average grain size of $1.37 \mu \mathrm{m}$ distributed within a wide size region. Fig. 1(c) shows the XRD patterns of the KNN-5LS-4BNZS. The obtained ceramics exhibited pure perovskite structure without any secondary phase.

Fig. 2(a) shows the ferroelectric hysteresis loops of the KNN5LS-4BNZS ceramics under the electric field of $3 \mathrm{kV} \mathrm{mm}^{-1}$ by heating from room temperature up to $200{ }^{\circ} \mathrm{C}$. The maximum polarization $P_{\max }$ and remnant polarization $P_{\mathrm{r}}$ changed slightly with $\Delta P_{\max }$ of $7 \%$ and $\Delta P_{\mathrm{r}}$ of $18 \%$ respectively in the wide temperature range. It indicates that the switched polarization state along the direction of the external electric field was of little variation. As another important characteristic for ferroelectric property, the coercive field $\left(E_{\mathrm{c}}\right)$ which indicates the energy barrier between the equivalent states of the order parameter decreased on increasing temperature, i.e. when getting closer to its Curie temperature around $270{ }^{\circ} \mathrm{C}$. Although the difference of $32 \%$ in $E_{\mathrm{c}}$, the domain switching behavior with similar $P_{\max }$ and $P_{\mathrm{r}}$ under comparable large electric field may benefit the temperature stability for domain-switching related applications.

Fig. 3(a) shows the electrostrain behavior in the temperature range from $20{ }^{\circ} \mathrm{C}$ to $200{ }^{\circ} \mathrm{C}$. All the electrostrain curves show the typical butterfly shape. Different from the hysteresis loops, which involved all the domain switching, the electrostrain curves only reflected the population of non- $180^{\circ}$ domain switching. For ferroelectrics, the non $-180^{\circ}$ polarization switching is always coupled to the lattice strain, an instability in polarization orientation (i.e., isotropy of polarization) will 

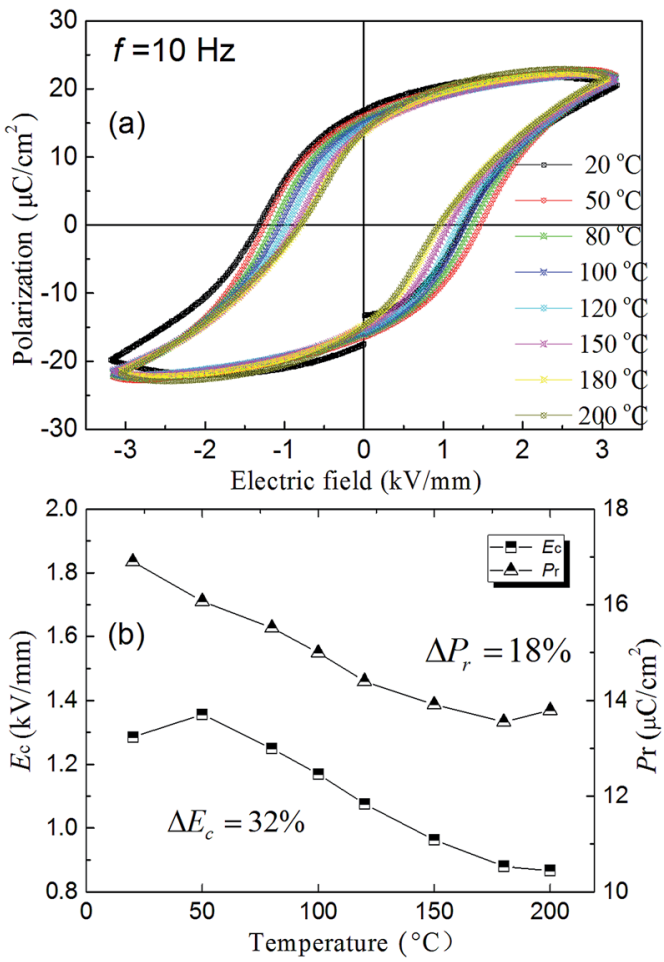

Fig. 2 (a) Ferroelectric hysteresis loops of the KNN-5LS-4BNZS ceramics from room temperature to $200^{\circ} \mathrm{C}$. (b) Variation of the $P_{r}$ and $E_{\mathrm{c}}$ of the KNN-5LS-4BNZS ceramics with the increasing temperature.

necessarily induce a softening in elastic modulus, and hence a high electrostrain. Therefore, low polarization anisotropy and elastic softening go hands in hands with each other, both contributing to a high electrostrain. As summarized in Fig. 3(b), the $S_{\text {neg }}$ (the strain between minimum strain and strain under zero electric field) of the ceramics decreased. While the $S_{\text {pos }}$ (the strain between the maximum strain and the strain under zero electric field) showed little change with $16 \%$ in the temperature range from room temperature to $200{ }^{\circ} \mathrm{C}$. It consisted with the hysteresis loop behavior and may benefit the applications with high temperature stability. Such results can be understood by two aspects. Firstly the ceramics are considered to possess multi-phase boundary region at room temperature according to previous report which may greatly enhance the responds under small ac external field, no matter electrical or mechanical. On decreasing the temperature the tetragonality enhanced, i.e. the population of $90^{\circ} / 180^{\circ}$ domain increasing. Comparing with the $60^{\circ} / 120^{\circ}$ domain for $\mathrm{O}$ phase and $71^{\circ} / 109^{\circ}$ domain for $\mathrm{R}$ phase, such domains morphology in tetragonal phase involves little

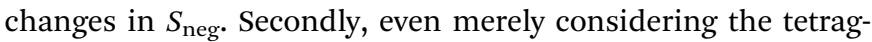
onal phase the tetragonality $c / a$ decreased when approaching its $T_{\mathrm{c}}$; it may consequently decrease the $S_{\mathrm{neg}}$. At room temperature the total electrostrain of $0.15 \%$ under the electric field of $3 \mathrm{kV}$ $\mathrm{mm}^{-1}$ can be expressed by the converse piezoelectric coefficient $d_{33}^{*}$ of $500 \mathrm{pm} \mathrm{V}^{-1}\left(d_{33}^{*}=S_{\max } / E_{\max }\right.$, where $S_{\max }$ and $E_{\max }$ denote the maximum strain and the maximum electric field strength, respectively) under the bipolar strain from the unpoled ceramics. It is superior the normal $\mathrm{KNbO}_{3}$ based ceramics with $d_{33}^{*}$ of $100-200 \mathrm{pm} \mathrm{V}^{-1}$.
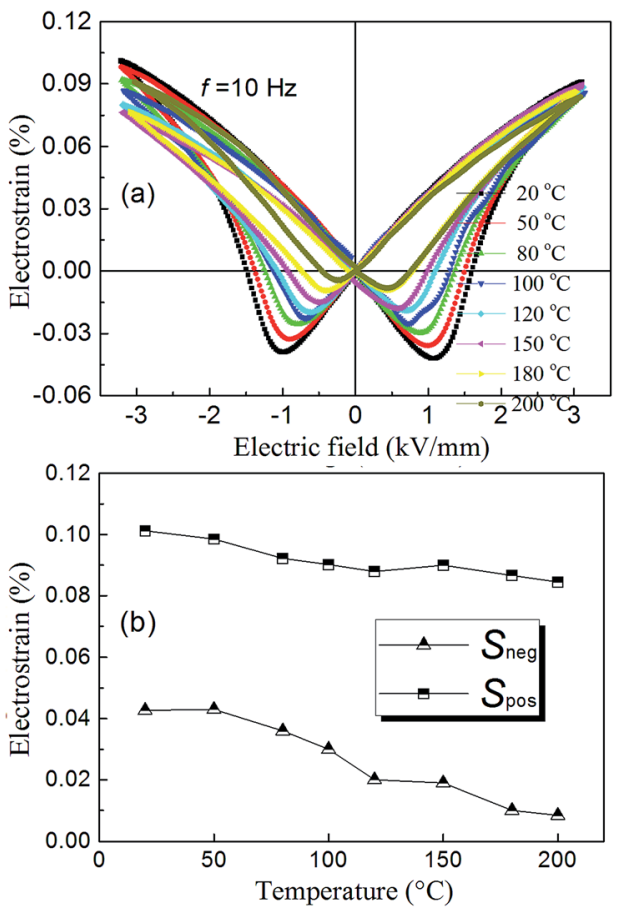

Fig. 3 (a) Electrostrain loops of the KNN-5LS-4BNZS ceramics from room temperature to $200{ }^{\circ} \mathrm{C}$. (b) Variation of the $S_{\text {neg }}$ and $S_{\text {pos }}$ of the KNN-5LS-4BNZS ceramics with increasing temperature.

More interestingly, large electrostrain over $0.24 \%$ under the electric field of $3 \mathrm{kV} \mathrm{mm}^{-1}$ can be achieved after poling treatment. Here the ceramic sample was poled at room temperature under the electric field of $5 \mathrm{kV} \mathrm{mm}^{-1}$ for $10 \mathrm{~min}$. Previous study has reported the excellent electrostrain behaviors in the KNNbased ceramics; CZ5 $\left(0.95\left(\mathrm{Na}_{0.19} \mathrm{~K}_{0.49} \mathrm{Li}_{0.02}\right)\left(\mathrm{Nb}_{0.8} \mathrm{Ta}_{0.2}\right) \mathrm{O}_{3^{-}}\right.$ $0.05 \mathrm{CaZrO}_{3}$ ) reported by $\mathrm{Wang}^{20}$ exhibited the strain of $0.16 \%$ at $6 \mathrm{kV} \mathrm{mm}{ }^{-1}$ and textured LF4 reported by Saito showed the electrostrain of $0.17 \%$ at $2 \mathrm{kV} \mathrm{mm}^{-1}$ (while untextured showed strain of only $0.085 \%$ at $\left.2 \mathrm{kV} \mathrm{mm}{ }^{-1}\right){ }^{12}$ The KNN-5LS-4BNZT ceramics showed even more promising performance at room temperature.

Fig. 4(b) shows the comparison of the strain behavior in wide temperature range between the KNN-5LS-4BNZT ceramics and other high-performed piezoelectric ceramics, including PZT4 ceramics, which is famous for its high electrostrain and good temperature stability. Here the strain behavior is characterized by the normalized piezoelectric coefficient $d_{33}^{*}$. We concluded that the $d_{33}^{*}$ of the KNN-5LS-4BNZT ceramics exceed that of the CZ5 and Bi-based lead-free ceramics in wide temperature range. Even more, it can rival the most widely used PZT4 ceramics and the textured LF4 which suffered a very complex texturing technology. Besides, such electrostrain exhibited excellent temperature stability with $\Delta \varepsilon$ of $3 \%$ in the wide temperature range from room temperature to $200{ }^{\circ} \mathrm{C}$, which is even superior to the PZT4 with $\Delta \varepsilon$ of $15 \%$ and textured LF4 $\Delta \varepsilon$ of $7 \%$. From Fig. 4(a) we notice that KNN-5LS-4BNZT exhibited small hysteresis in the strain-electric field curves besides the good electrostrain behavior, which can be defined as the area within the strain curves. Such small hysteresis possess two advantages, one is the 

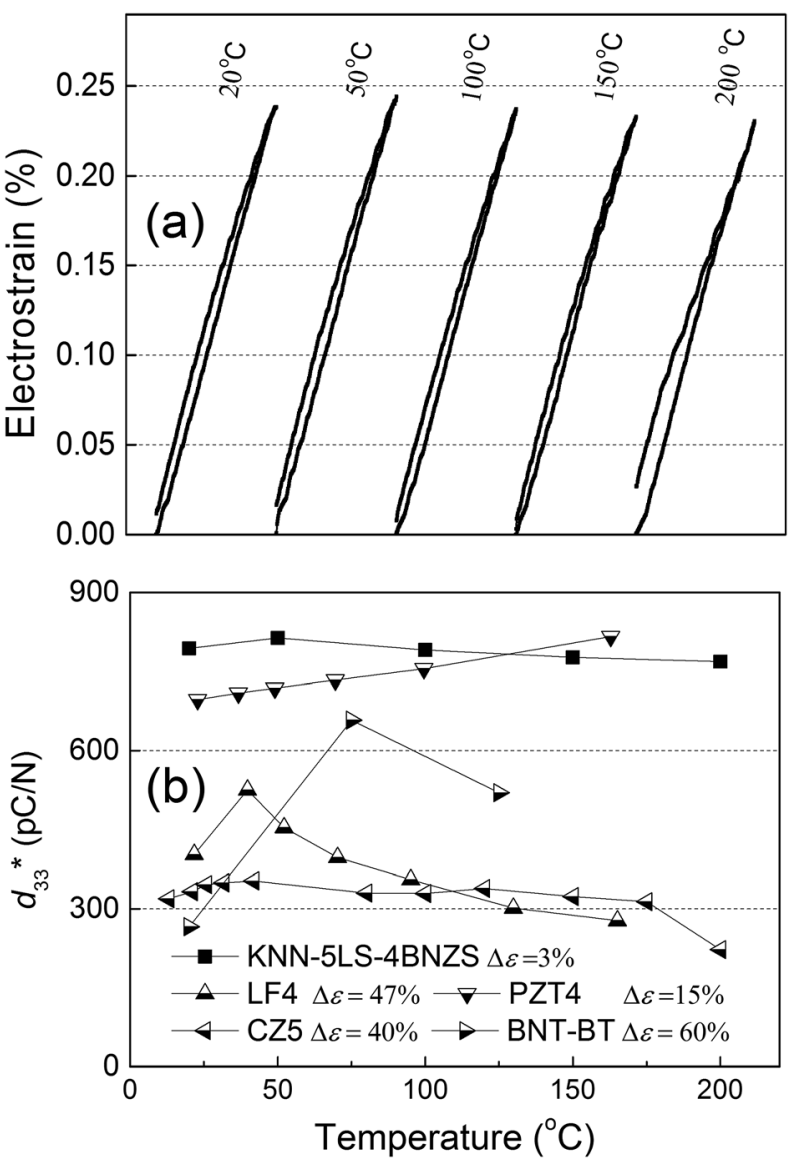

Fig. 4 (a) Electrostrain loops of the poled KNN-5LS-4BNZS ceramics from room temperature to $200^{\circ} \mathrm{C}$. (b) Strain behaviors of temperature range from $20^{\circ} \mathrm{C}$ to $200^{\circ} \mathrm{C}$ of the KNN-5LS-4BNZS, LF4, PZT4, CZ5, BNT-BT ceramics.

high accuracy between the forward and the backward processes while the other is the small energy loss when cycling. Additionally, the present KNN-5LS-4BNZT exhibited a large $d_{33}$ of $207 \mathrm{pC} \mathrm{N}^{-1}$. It is also promising for lead-free piezoelectric material which may trigger the applications under small signal, e.g. the sensors.

\section{Conclusions}

In summary, a new electro-shape-memory material with great strain behavior and temperature stability KNN-5LS-4BNZS ceramic has been synthesized by conventional solid-state reaction method. A unipolar strain of $0.24 \%$ with a slim electrostrain curve under an electric field of $3 \mathrm{kV} \mathrm{mm}^{-1}$ is achieved after poled. Furthermore, a variation of only $3 \%$ of the strain behavior is obtained when heating to $200{ }^{\circ} \mathrm{C}$. Such splendid performances can be applied to actuators with the demands of high precision and good thermal stability and surely challenge the current mainstream lead-based material PZT4. We believe the coexistence of the ferroelectric phases may attribute to the excellent strain behavior of the samples and the small variations of $P_{\mathrm{r}}$ and $S_{\text {pos }}$ with increasing temperature would help to explain the steady thermal performance of the ceramic.

\section{Acknowledgements}

The authors acknowledge the support of National Nature Science Foundation of China (51422704) and State Key of Laboratory of Electrical Insulation and Power Equipment, Xi'an Jiaotong University (EIPE14113).

\section{Notes and references}

1 L. X. Zhang and X. Ren, Mater. Sci. Eng., A, 2006, 438, 354.

2 K. Bhattacharya and G. Ravichandran, Acta Mater., 2003, 51, 5941.

3 K. Uchino, Am. Ceram. Soc. Bull., 1986, 65, 647.

4 B. Jaffe, Piezoelectric Ceramics, Academic Press, London, 1971.

5 L. E. Cross, S. J. Jang, R. E. Newnham, S. Nomura and K. Uchino, Ferroelectrics, 1980, 23, 187.

6 Y. Dai, Y. Chen, H. Shen, Z. Zhang and Y. Wang, Ferroelectrics, 2001, 251, 77.

7 S. E. Park and T. R. Shrout, J. Appl. Phys., 1997, 82, 1804.

8 S. T. Zhang, A. B. Kounga, W. Jo, C. Jamin, K. Seifert, T. Granzow, J. Rodel and D. Damjanovic, Adv. Mater., 2009, 21, 4716.

9 Z. Yu, C. Ang, R. Guo and A. S. Bhalla, Appl. Phys. Lett., 2002, 92, 1489.

10 L. X. Zhang, W. Chen and X. Ren, Appl. Phys. Lett., 2004, 85, 5658.

11 W. F. Liu, W. Chen, L. Yang, L. X. Zhang, Y. Wang, C. Zhou, S. T. Li and X. Ren, Appl. Phys. Lett., 2006, 89, 172908.

12 Y. Saito, H. Takao, T. Tani, T. Nonoyama, K. Takatori, T. Homma, T. Nagaya and M. Nakamura, Nature., 2004, 432, 84.

13 J. G. Wu, D. Q. Xiao and J. G. Zhu, Chem. Rev., 2015, 115, 2559.

14 X. Lv, J. G. Wu, D. Q. Xiao, H. Tao, Y. Yuan, J. G. Zhu, X. J. Wang and X. J. Lou, Dalton Trans., 2015, 44, 4440.

15 X. P. Wang, J. G. Wu, D. Q. Xiao, X. J. Cheng, T. Zheng, X. J. Lou, B. Y. Zhang and J. G. Zhu, ACS Appl. Mater. Interfaces, 2014, 6, 6177.

16 H. Tao, J. G. Wu, D. Q. Xiao, J. G. Zhu, X. P. Wang and X. J. Lou, ACS Appl. Mater. Interfaces, 2014, 6, 20358.

17 T. Zheng, J. G. Wu, X. J. Cheng, X. P. Wang, B. Y. Zhang, D. Q. Xiao, J. G. Zhu, X. J. Wang and X. J. Lou, J. Mater. Chem. C, 2014, 2, 8796.

18 K. Xu, J. Li, X. Lv, J. Wu, X. Zhang, D. Xiao and J. Zhu, Adv. Mater., 2016, 28, 8519.

19 B. Wu, H. Wu, J. Wu, D. Xiao, J. Zhu and S. J. Pennycook, J. Am. Chem. Soc., 2016, 138, 15459.

20 K. Wang, F. Z. Yao, W. Jo, D. Gobeljic, V. V. Shvartsman, D. C. Lupascu, J. F. Li and J. Rödel, Adv. Funct. Mater., 2013, 23, 4079.

21 Y. G. Yao, C. Zhou, D. C. Lv, D. Wang, H. J. Wu, Y. D. Yang and X. B. Ren, Europhys. Lett., 2012, 98, 27008.

22 J. Rödel, W. Jo, K. T. P. Seifert, E. Anton, T. Granzow and D. Damjanovic, J. Am. Ceram. Soc., 2009, 92, 1153. 
23 M. Acosta, N. Khakpash, T. Someya, N. Novak, W. Jo, H. Nagata, G. A. Rossetti Jr and J. Rödel, Phys. Rev. B: Condens. Matter Mater. Phys., 2015, 91, 104108.

24 A. A. Heitmann and G. A. Rossetti, J. Am. Ceram. Soc., 2014, 97, 1661.

25 W. F. Rao and Y. U. Wang, Appl. Phys. Lett., 2008, 92, 102905.

26 W. F. Rao and Y. U. Wang, Appl. Phys. Lett., 2007, 91, 052901.

27 X. P. Wang, J. G. Wu, X. J. Cheng, B. Y. Zhang, J. G. Zhu and D. Q. Xiao, Ceram. Int., 2013, 39, 8021.
28 J. Wu, D. Xiao and J. Zhu, J. Mater. Sci.: Mater. Electron., 2015, 26, 9297.

29 R. Thomas, V. K. Varadan, S. Komarneni and D. C. Dube, J. Appl. Phys., 2001, 90, 1480.

30 W. F. Liu, S. Tsukada and Y. Akishige, Funct. Mater. Lett., 2014, 7, 599.

31 A. Dixit, S. B. Majumder, R. S. Katiyar and A. S. Bhalla, Appl. Phys. Lett., 2003, 82, 2679.

32 D. Damjanovic, Hysteresis in Piezoelectric and Ferroelectric Materials, Academic Press-Elsevier, New York, 2006. 